\title{
Three-flavoured neutrino oscillations and the Leggett-Garg inequality
}

\author{
Debashis Gangopadhyay ${ }^{\mathrm{a}}$, Animesh Sinha Roy ${ }^{\mathrm{b}}$ \\ Department of Physics, Ramakrishna Mission Vivekananda University, Belur Matth, Howrah, West Bengal 711202, India
}

Received: 16 February 2017 / Accepted: 13 April 2017 / Published online: 25 April 2017

(C) The Author(s) 2017. This article is an open access publication

\begin{abstract}
Three-flavoured neutrino oscillations are investigated in the light of the Leggett-Garg inequality (LGI). The results obtained are: (a) The maximum violation of the LGI is 2.17036 for neutrino path length $L_{1}=140.15 \mathrm{~km}$ and $\Delta L=1255.7 \mathrm{~km}$. (b) The presence of the mixing angle $\theta_{13}$ enhances the maximum violation of LGI by $4.6 \%$. (c) The currently known mass hierarchy parameter $\alpha=0.0305$ increases the maximum violation of LGI by $3.7 \%$. (d) The presence of a CP-violating phase parameter enhances the maximum violation of LGI by $0.24 \%$, thus providing an alternative indicator of $C P$ violation in three-flavoured neutrino oscillations. The outline of an experimental proposal is suggested whereby the findings of this investigation may be verified.
\end{abstract}

\section{Introduction}

The Leggett-Garg inequality (LGI) [1] is useful to test the quantumness of a system through successive measurement outcomes at different times on the same system. In a previous work [2] we showed that two-state neutral kaon oscillations and two-state neutrino oscillations are quantum phenomena by demonstrating that the LGI is violated in both cases.

Note that the kaon and neutrino cases comprised two different kinds of two-state quantum systems. Oscillations between $K^{0}$ and $\bar{K}^{0}$ states indicate a decaying two-state oscillating quantum system. On the other hand, neutrino oscillations between the two-flavour eigenstates $v_{e}$ and $v_{\mu}$ signify a conservative two-state quantum system. In [2] for a decaying kaon system, the maximum violation of LGI in the presence of $\mathrm{CP}$ violation is when the correlator $C=2.36463$

\footnotetext{
a e-mail: debashis@ rkmvu.ac.in

b e-mail: animesh.roy@rkmvu.ac.in
}

(defined below in Sect. 2), while in the absence of CP violation the LGI violation is maximum when $C=2.36448$. This is significantly smaller than the Tsirelson bound for the LGI in two-state system given by $C_{\text {Tsirelson }}=2 \sqrt{2}=2.82843$. In the case of conservative two-flavour neutrino oscillations the maximum violation of LGI is when $C=2.76000$. Similar work has also been done in two-state neutrino oscillations [3]. There the authors have demonstrated how oscillation phenomena can be used to test for violations of the classical bound by performing measurements on an ensemble of neutrinos at distinct energies.

The existence of neutrino mass has been a subject of keen interest over the last 50 years [4-6]. In 2001 the third generation of neutrinos (tau neutrino) was discovered by the DONUT collaboration [7]. Exhaustive details regarding various aspects of neutrino masses and oscillations can be found in $[8,9]$ and the references therein. The next investigation, therefore, logically should be the LGI in the scenario of threeflavoured neutrino oscillations, both without and with $\mathrm{CP}$ violation. This is what we set out to accomplish in the present work. The effect of $\mathrm{CP}$ violation for three-flavoured neutrino oscillations may stimulate further investigations in this area. We also consider matter interactions with the neutrino. Here we have analysed the LGI in the context of two small parameters, viz. the sine of the mixing angle $\theta_{13}, \sin \theta_{13}<<1$ and the mass hierarchy parameter $\alpha<<1$. Note that the mixing angles are Eulerian angles relating the set $\left(v_{e}, v_{\mu}, v_{\tau}\right)$ to the mass eigenstates $\left(v_{1}, v_{2}, v_{3}\right)$ in the relevant space as shown in Fig. 1, while $\alpha \equiv \frac{\Delta m_{21}^{2}}{\Delta m_{31}^{2}}$ with $\Delta m_{21}^{2} \equiv m_{2}^{2}-m_{1}^{2}$, $\Delta m_{31}^{2} \equiv m_{3}^{2}-m_{1}^{2}$, where $m_{i}, i=1,2,3$, denotes the mass of the $i$ th species of neutrino.

In Sect. 2, we give a brief introduction of LGI. In Sect. 3 we discuss the three-flavoured neutrino oscillations. In Sect. 4 the LGI is evaluated and analysed. In Sect. 5 an outline is given of how one can actually experimentally verify the LGI in three-flavoured neutrino oscillations. Section 6 summarises our results. Appendix is in Sect. 7. 


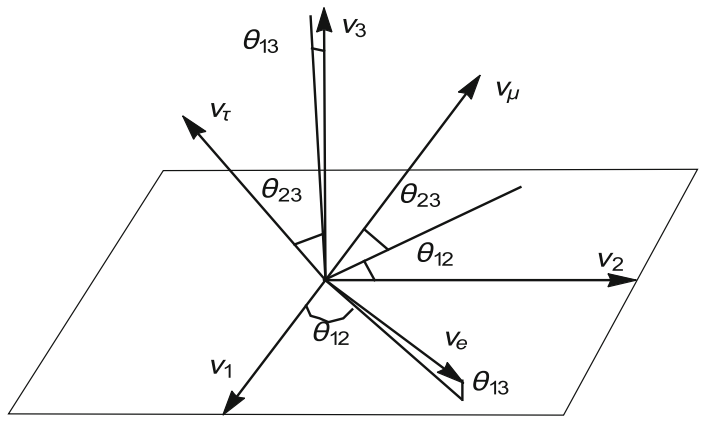

Fig. 1 Neutrino mixing angles without $\mathrm{CP}$ violation as Euler angles relating $\left(v_{e}, v_{\mu}, v_{\tau}\right)$ to the mass eigenstates $\left(v_{1}, v_{2}, v_{3}\right)$

\section{Leggett-Garg inequality}

Bell's inequality (BI) [10] is based on the assumption of local realism - an intrinsic property of classical physics. Violation of local realism signifies quantum phenomena. BI is a testable algebraic inequality constructed from certain combination of correlation functions for the outcomes of an observable quantity measurement on two spatially separated systems at the same instant of time. BI is violated by quantum physics in the presence of quantum entanglement between two spatially separated systems and implies that the quantum world is nonlocal [11-16]. Leggett and Garg [1] constructed another algebraic inequality based on the assumption of macrorealism in terms of time separated correlation functions corresponding to the successive measurement outcomes at different times on a single system.

The assumptions underlying the LGI [1] are macroscopic realism (MR) and noninvasive measurability (NIM). MR means that a macroscopic system during its time evolution, is (at any instant time) in a definite one of the available states. NIM means it is possible in principle to determine which of the states the system is in, without affecting the states themselves or the system's subsequent dynamics. These two aspects together constitute macrorealism.

Consider a two-state system and an observable quantity $Q(t)$ such that whenever $Q(t)$ is measured it takes values +1 or -1 depending on whether it is in state 1 or state 2 , respectively. Next consider a collection of runs starting from identical initial conditions such that in the first set of runs $Q$ is measured at times $t_{1}$ and $t_{2}$; in the second at $t_{2}$ and $t_{3}$; in the third at $t_{3}$ and $t_{4}$; in the fourth at $t_{1}$ and $t_{4}\left(t_{1}<t_{2}<t_{3}<t_{4}\right)$. From such measurements it is straightforward to determine the temporal correlation function $C_{i j} \equiv\left\langle Q\left(t_{i}\right) Q\left(t_{j}\right)\right\rangle$. Any physical system obeying the assumptions of a macrorealistic theory will then give the Leggett-Garg inequality [1]:

$C \equiv C_{12}+C_{23}+C_{34}-C_{14} \leq 2$.

A wide range of quantum systems violate the upper bound of the LGI. This allows one to use the LGI to probe quan- tum mechanics $(\mathrm{QM})$ in the macroscopic regime [17-34]. A detailed review on LGI is given in [35].

The Legget-Garg Inequlity involves the time parameter whereas the relevant probabilities (given below in Sect. 3 onwards) are expressed in terms of the base line length parameter $L$. But $L=c t, c$ is the velocity of light. So $t$ is automatically present. Now the correlations in time are transcribed into correlations in length.

Consider an $n$-state system. As before, measurements of a macroscopic property $Q$ can yield only two values \pm 1 , i.e. $Q$ is a dichotomic variable. If some states (say $k$ states where $k<n)$ take the value +1 then all the remaining $n-k$ states will take the value -1 . This is no problem because states with the same value of $Q$ may be considered as microscopically distinct states with the same macroscopic property $Q$. MR and NIM then imply that the system has a definite value of $Q$ at all times and this value is independent of previous measurements on the system. Therefore, the bound for Eq. (1) in macrorealistic theories remains the same.

We now consider LGI in the three-flavoured neutrino oscillations.

\section{Three-flavoured neutrino oscillations}

During propagation neutrinos undergo oscillations between the three-flavoured eigenstates $v_{e}, v_{\mu}$ and $v_{\tau}$. Consider the standard parameterisation of the Pontecorvo-MakiNakagawa-Sakata (PMNS) matrix $U$ that mixes the three neutrino flavour states $[36,37]$ :

$$
\begin{aligned}
& U= \\
& \left(\begin{array}{lll}
c_{12} c_{13} & s_{12} c_{13} & s_{13} e^{-i \delta_{\mathrm{CP}}} \\
-s_{12} c_{23}-c_{12} s_{13} s_{23} e^{i \delta_{\mathrm{CP}}} & c_{12} c_{23}-s_{12} s_{13} s_{23} e^{i \delta_{\mathrm{CP}}} & c_{13} s_{23} \\
s_{12} s_{23}-c_{12} s_{13} c_{23} e^{i \delta_{\mathrm{CP}}} & -c_{12} s_{23}-s_{12} s_{13} c_{23} e^{i \delta_{\mathrm{CP}}} & c_{13} c_{23}
\end{array}\right)
\end{aligned}
$$

where $\theta_{i j}$ are the mixing angles, $c_{i j} \equiv \cos \theta_{i j}, s_{i j} \equiv \sin \theta_{i j}$ and $\delta_{\mathrm{CP}}$ is the Dirac-type CP-violating phase. If $P_{\alpha \beta} \equiv$ $P\left(v_{\alpha} \rightarrow v_{\beta}\right)$ is the transition probability from one neutrino flavour $\alpha$ to another flavour $\beta$, then in general the functional dependence of $P_{\alpha \beta}$ is

$P_{\alpha \beta}=P_{\alpha \beta}\left(\Delta m_{21}^{2}, \Delta m_{31}^{2}, \theta_{12}, \theta_{13}, \theta_{23}, \delta_{\mathrm{CP}}, E, L, V(x)\right)(3)$ where $\alpha, \beta \equiv e, \mu, \tau$. Here $\Delta m_{i j}^{2} \equiv m_{i}^{2}-m_{j}^{2}$ with $m_{i}$ being the mass of the $i$ th species. $E$ is the neutrino energy, $L$ is the baseline length, and $V(x)$ is the matter-induced effective potential, $x \in[0, L]$ is the coordinate along the neutrino path.

$\Delta m_{i j}^{2}, \theta_{i j}$ 's and $\delta_{\mathrm{CP}}$ are fundamental parameters and the same for all experiments. On the other hand $E, L$ and $V$ vary from experiment to experiment.

In [38] complete sets of series expansion formulae for neutrino oscillation probabilities in matter of constant density 
have been calculated taking into account the three flavours. We will consider the neutrino energies of the order of 1 $\mathrm{GeV}$. Therefore we consider the appropriate double expansion given in [38] up to the second order in both mass hierarchy parameter $\alpha \equiv \frac{\Delta m_{21}^{2}}{\Delta m_{31}^{2}}$ and $s_{13}=\sin \theta_{13}$.

Let us start with an electron-neutrino beam at time $t=0$, i.e. $L=0$. After time $t$, i.e. distance $L=c t$, the probability of finding $v_{e}, v_{\mu}$ and $v_{\tau}$ are, respectively [38],

$$
\begin{aligned}
& P_{\nu_{e}}= 1-\alpha^{2} \sin ^{2} 2 \theta_{12} \frac{\sin ^{2}\left(\frac{V L}{2}\right)}{\left(\frac{2 E V}{\Delta m_{31}^{2}}\right)^{2}} \\
&-4 s_{13}^{2} \frac{\sin ^{2}\left\{\left(\frac{2 E V}{\Delta m_{31}^{2}}-1\right) \frac{\Delta m_{31}^{2} L}{4 E}\right\}}{\left(\frac{2 E V}{\Delta m_{31}^{2}}-1\right)^{2}}, \\
& P_{\nu_{\mu}}= \alpha^{2} \sin ^{2} 2 \theta_{12} c_{23}^{2} \frac{\sin ^{2}\left(\frac{V L}{2}\right)}{\left(\frac{2 E V}{\Delta m_{31}^{2}}\right)^{2}}+4 s_{13}^{2} s_{23}^{2} \\
& \times \frac{\sin ^{2}\left\{\left(\frac{2 E V}{\Delta m_{31}^{2}}-1\right) \frac{\Delta m_{31}^{2} L}{4 E}\right\}}{\left(\frac{2 E V}{\Delta m_{31}^{2}}-1\right)^{2}}+2 \alpha s_{13} \sin 2 \theta_{12} \sin 2 \theta_{23} \\
& \times \cos \left(\frac{\Delta m_{31}^{2} L}{4 E}-\delta_{\mathrm{CP}}\right) \frac{\sin \left(\frac{V L}{2}\right)}{\left(\frac{2 E V}{\Delta m_{31}^{2}}\right)} \frac{\sin \left\{\left(\frac{2 E V}{\Delta m_{31}^{2}}-1\right) \frac{\Delta m_{31}^{2} L}{4 E}\right\}}{\left(\frac{2 E V}{\Delta m_{31}^{2}}-1\right)}
\end{aligned}
$$

$$
\begin{aligned}
& P_{\nu_{\tau}}=\alpha^{2} \sin ^{2} 2 \theta_{12} s_{23}^{2} \frac{\sin ^{2}\left(\frac{V L}{2}\right)}{\left(\frac{2 E V}{\Delta m_{31}^{2}}\right)^{2}}+4 s_{13}^{2} c_{23}^{2} \\
& \times \frac{\sin ^{2}\left\{\left(\frac{2 E V}{\Delta m_{31}^{2}}-1\right) \frac{\Delta m_{31}^{2} L}{4 E}\right\}}{\left(\frac{2 E V}{\Delta m_{31}^{2}}-1\right)^{2}}-2 \alpha s_{13} \sin 2 \theta_{12} \sin 2 \theta_{23} \\
& \times \cos \left(\frac{\Delta m_{31}^{2} L}{4 E}-\delta_{\mathrm{CP}}\right) \frac{\sin \left(\frac{V L}{2}\right)}{\left(\frac{2 E V}{\Delta m_{31}^{2}}\right)} \frac{\sin \left\{\left(\frac{2 E V}{\Delta m_{31}^{2}}-1\right) \frac{\Delta m_{31}^{2} L}{4 E}\right\}}{\left(\frac{2 E V}{\Delta m_{31}^{2}}-1\right)} .
\end{aligned}
$$

So it is easy to say that after travelling the distance $L$, the probability of obtaining $v_{e}, v_{\mu}$ and $v_{\tau}$ are given by Eqs. (4), (5) and (6), respectively. The joint probability of finding the neutrino with flavours $v_{e}$ and $v_{\mu}$ after travelling the respective distances $L_{1}$ and $L_{2}\left(L_{2}>L_{1}\right)$ is then

$$
\begin{gathered}
P_{v_{e}, v_{\mu}}\left(L_{1}, L_{2}\right)=P_{v_{e}}\left(L_{1}\right) \times P_{v_{\mu}}\left(L_{2}-L_{1}\right) \\
=\left[1-\alpha^{2} \sin ^{2} 2 \theta_{12} \frac{\sin ^{2}\left(\frac{V L_{1}}{2}\right)}{\left(\frac{2 E V}{\Delta m_{31}^{2}}\right)^{2}}-4 s_{13}^{2}\right.
\end{gathered}
$$

$$
\begin{aligned}
& \left.\times \frac{\sin ^{2}\left\{\left(\frac{2 E V}{\Delta m_{31}^{2}}-1\right) \frac{\Delta m_{31}^{2} L_{1}}{4 E}\right\}}{\left(\frac{2 E V}{\Delta m_{31}^{2}}-1\right)^{2}}\right]\left[\alpha^{2} \sin ^{2} 2 \theta_{12} c_{23}^{2} \frac{\sin ^{2}\left(\frac{V\left(L_{2}-L_{1}\right)}{2}\right)}{\left(\frac{2 E V}{\Delta m_{31}^{2}}\right)^{2}}\right. \\
& +4 s_{13}^{2} s_{23}^{2} \frac{\sin ^{2}\left\{\left(\frac{2 E V}{\Delta m_{31}^{2}}-1\right) \frac{\Delta m_{31}^{2}\left(L_{2}-L_{1}\right)}{4 E}\right\}}{\left(\frac{2 E V}{\Delta m_{31}^{2}}-1\right)^{2}}+2 \alpha s_{13} \sin 2 \theta_{12} \\
& \times \sin 2 \theta_{23} \cos \left(\frac{\Delta m_{31}^{2}\left(L_{2}-L_{1}\right)}{4 E}-\delta_{\mathrm{CP}}\right) \frac{\sin \left(\frac{V\left(L_{2}-L_{1}\right)}{2}\right)}{\left(\frac{2 E V}{\Delta m_{31}^{2}}\right)} \\
& \left.\times \frac{\sin \left\{\left(\frac{2 E V}{\Delta m_{31}^{2}}-1\right) \frac{\Delta m_{31}^{2}\left(L_{2}-L_{1}\right)}{4 E}\right\}}{\left(\frac{2 E V}{\Delta m_{31}^{2}}-1\right)}\right] .
\end{aligned}
$$

Similarly one can find the other eight joint probabilities:

$P_{v_{e}, v_{e}}\left(L_{1}, L_{2}\right), \quad P_{v_{e}, v_{\tau}}\left(L_{1}, L_{2}\right), \quad P_{v_{\mu}, v_{e}}\left(L_{1}, L_{2}\right), \quad P_{v_{\mu}, v_{\mu}}$ $\left(L_{1}, L_{2}\right), P_{v_{\mu}, v_{\tau}}\left(L_{1}, L_{2}\right), P_{v_{\tau}, v_{e}}\left(L_{1}, L_{2}\right), P_{v_{\tau}, v_{\mu}}\left(L_{1}, L_{2}\right)$, $P_{v_{\tau}, v_{\tau}}\left(L_{1}, L_{2}\right)$. The transition probabilities required to evaluate the above joint probabilities are given in detail in [38].

\section{Evaluating and analysing LGI for three flavours of neutrino}

In the three-flavoured neutrino oscillations, we assume that the dichotomic observable $Q$ takes the value +1 when the system is found in the electron-neutrino flavour state $v_{e} . Q$ takes the value -1 if the system is found in any one of the muon neutrino $v_{\mu}$ or tau neutrino $v_{\tau}$ states. Then the correlation function $C_{12}$ can be evaluated by using all the 9 joint probabilities as

$$
\begin{aligned}
C_{12}= & \left\langle Q\left(L_{1}\right) Q\left(L_{2}\right)\right\rangle \\
= & P_{v_{e}, v_{e}}\left(L_{1}, L_{2}\right)-P_{v_{e}, v_{\mu}}\left(L_{1}, L_{2}\right)-P_{v_{e}, v_{\tau}}\left(L_{1}, L_{2}\right) \\
& -P_{v_{\mu}, v_{e}}\left(L_{1}, L_{2}\right)+P_{v_{\mu}, v_{\mu}}\left(L_{1}, L_{2}\right)+P_{v_{\mu}, v_{\tau}}\left(L_{1}, L_{2}\right) \\
& -P_{v_{\tau}, v_{e}}\left(L_{1}, L_{2}\right)+P_{v_{\tau}, v_{\mu}}\left(L_{1}, L_{2}\right)+P_{v_{\tau}, v_{\tau}}\left(L_{1}, L_{2}\right) .
\end{aligned}
$$

The exact expression of $C_{12}$ is given in the appendix. An interesting point in the expression for $C_{12}$ is that for a neutrino beam with given energy the correlation $C_{12}$ shows dependence on $L_{1}$ as well as the spatial separation $\left(L_{2}-L_{1}\right)$. It is also important to note that in the case of two-flavoured neutrino oscillations the correlation function depends only on the spatial seperation $\left(L_{2}-L_{1}\right)$ [2]. The other correlation functions, viz., $C_{23}, C_{34}$ and $C_{14}$ can be calculated in the same way and they exhibit similar features. Next one can evaluate the correlation function $C$ defined in Eq. (1) in order to study the maximum violation of LGI for three-flavoured neutrino oscillations. Varying the spatial separations, it is found that the maximum value of $C$ is attained essentially when all the spatial separations are taken to be same, i.e. $\left(L_{4}-L_{3}\right)=\left(L_{3}-L_{2}\right)=\left(L_{2}-L_{1}\right)=\Delta L$ and the cor- 


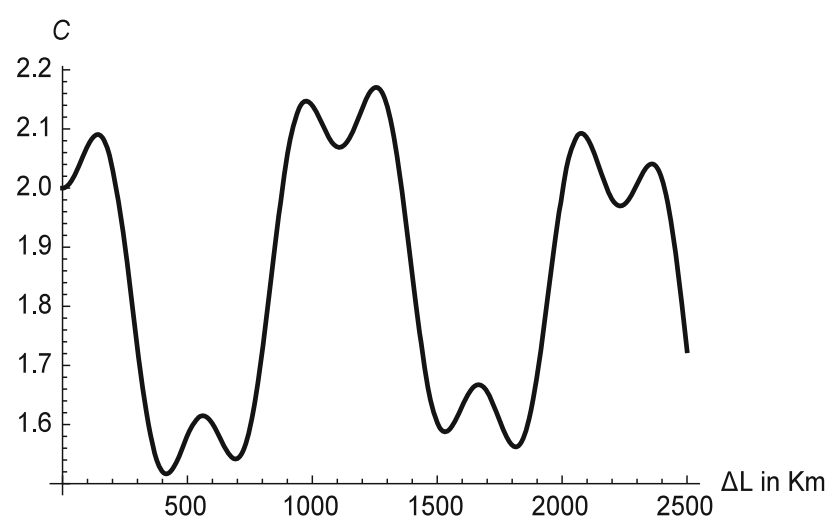

Fig. 2 Correlation $C$ as a function of $\Delta L$ in $\mathrm{km}$ for $L_{1}=140.15 \mathrm{~km}$. $C$ attains its maximum value 2.17036 at $\Delta L=1255.7 \mathrm{~km}$

relation function $C$ under this condition depends on $\Delta L$ and $L_{1}$.

We have calculated the maximum value of $C$ using the latest experimentally determined values given in [39]. These are $\Delta m_{21}^{2}=7.50 \times 10^{-5} \mathrm{eV}^{2}, \Delta m_{31}^{2}=2.457 \times 10^{-3} \mathrm{eV}^{2}$, $\theta_{12}=33.48^{\circ}, \theta_{23}=42.3^{\circ}, \theta_{13}=8.50^{\circ}, \delta_{\mathrm{CP}}=306^{\circ}$. Here the potential [38] $V=7.56 \times 10^{-14}\left(\frac{\rho}{\mathrm{g} / \mathrm{cm}^{3}}\right) Y_{e} \mathrm{eV}$, where $\rho$ is the matter density along the neutrino path and $Y_{e}$ is the number of electrons per nucleon. For terrestrial matter $Y_{e} \simeq 0.5$ [38]. For practical purposes taking $\rho$ constant is a very good approximation [40-42]. A typical value of the matter density is $\rho=3 \mathrm{~g} / \mathrm{cm}^{3}$ [38]. So the potential $V$ takes the value $V=11.34 \times 10^{-14} \mathrm{eV}$. Here we consider the energy of neutrino to be $1 \mathrm{GeV}$. Further, we consider various choices of $L_{1}$ and $\Delta L$ and find that the maximum value of $C$ reaches 2.17036 for $L_{1}=140.15 \mathrm{~km}$ and $\Delta L=1255.7 \mathrm{~km}$. It is important to note that the maximum QM violation of LGI in this case is significantly smaller than the maximum value of $C=2.76$ we calculated in [2] in the case of two-flavoured neutrino oscillations. For the given value of $L_{1}=140.15$ $\mathrm{km}$, the variation of the quantity $C$ with $\Delta L$ is shown in Fig. 2.

Now we investigate how the mixing angle $\theta_{13}$ affects the maximum value of $C$. If we put $\theta_{13}=0$, the maximum value of $C=2.07762$ for $L_{1}=638 \mathrm{~km}$ and $\Delta L=1376.34 \mathrm{~km}$. This is much lower than the actual value (2.17036) when $\theta_{13} \neq 0$. This value still belongs to the quantum domain because it is larger than 2 . So the presence of the mixing angle $\theta_{13}$ in three-flavour neutrino oscillations enhances the maximum violation of LGI by an amount of 0.09274 . If we increase $\theta_{13}$ from zero degree we see that the maximum value of the quantity $C$ also increases. This means increasing the mixing angle $\theta_{13}$ also increases the quantumness of the threeflavoured neutrino oscillations. The variation of $C$ with $\theta_{13}$ is shown in Fig. 3 below

One of the key parameters in three-flavour neutrino oscillations is the small mass hierarchy parameter $\alpha \equiv \frac{\Delta m_{21}^{2}}{\Delta m_{31}^{2}}$.

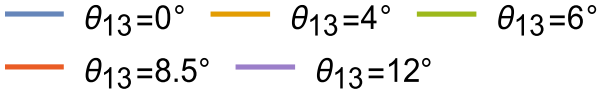

C
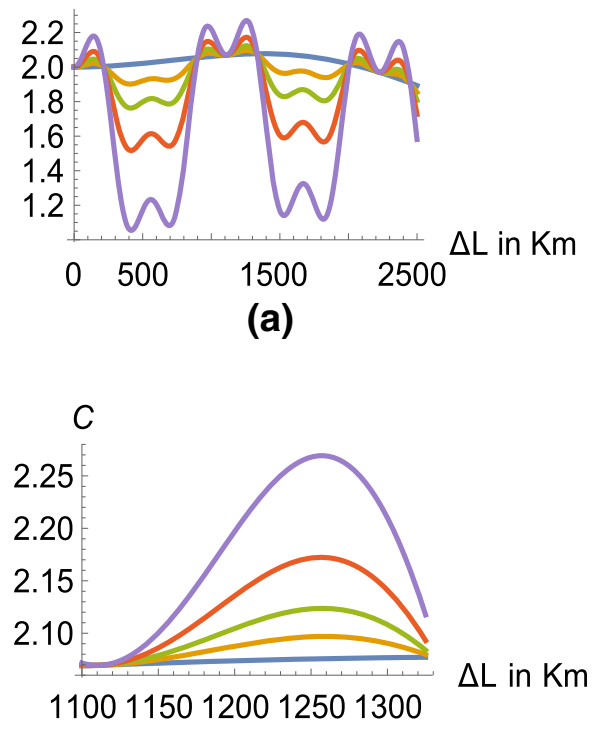

(b)

Fig. 3 a $C$ versus $\Delta L$ in $\mathrm{km}$ for different values of the mixing angle parameter $\theta_{13}$. Here $L_{1}=140.15 \mathrm{~km}$. Blue $0^{\circ}$, orange $4^{\circ}$, green $6^{\circ}$, red $8.5^{\circ}$ (actual experimentally measured value), violate $12^{\circ}$. b We focus around the region where the value of $C$ is maximum. The maximum value of $C$ increases with the increase of the value of the mixing angle $\theta_{13}$

In this section we investigate the dependence of $C$ on $\alpha$. If we put $\alpha=0$, i.e. $m_{1}=m_{2}$, the maximum value of $C$ becomes 2.09606 for $\Delta L=1252.74 \mathrm{~km}$. It is interesting to note that although now $m_{1}=m_{2}$ the maximum bound of the quantity $C$ is greater than 2, i.e., we are still in the quantum domain. For two-state neutrino oscillations [2], the condition $m_{1}=m_{2}$ implies that the maximum value of $C$ is 2, i.e. one is in the classical domain!. This is logical because this means there is only one neutrino mass, so there cannot be any oscillations. However, for three-state neutrino oscillations there are three neutrino masses and if two of them become equal then also there will exist possibility of neutrino oscillations because now there are effectively two masses. In the present case the presence of the non-zero value of $\alpha$ increases the maximum value of $C$ as shown in Fig. 4 . In Fig. 4 blue, orange, green and red color graphs correspond to the behaviour of the quantity $C$ for values of $\alpha=$ $0,0.01,0.0305$ (actual experimentally measured value) and 0.06 , respectively. For the present experimentally measured value of $\alpha$ the maximum value of the quantity $C$ increases by about $3.7 \%$. So the presence of non-zero $\alpha$ increases the quantumness in three-flavoured neutrino oscillations.

Next consider the effect of the $\mathrm{CP}$-violating phase parameter $\delta_{\mathrm{CP}}$ on the maximum value of $C$. If we ignore $\delta_{\mathrm{CP}}$ in 


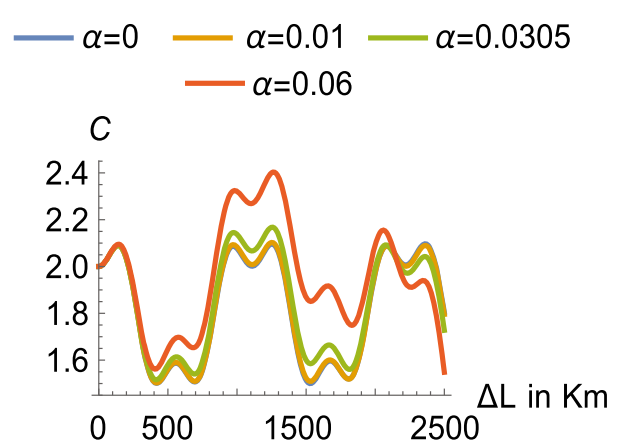

(a)

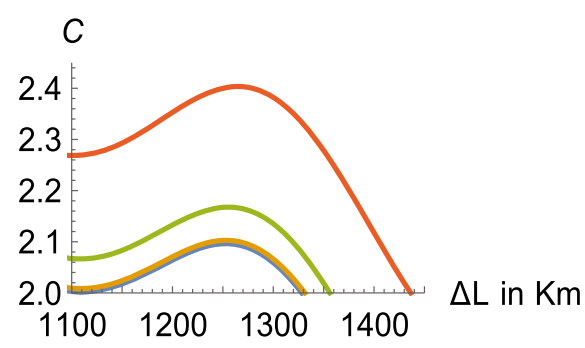

(b)

Fig. 4 Behaviour of the quantity $C$ with the variation of $\Delta L$ in $\mathrm{km}$ for different values of the mass hierarchy parameter $\alpha$ is shown in Fig. 4a. Here $L_{1}=140.15 \mathrm{~km}$. Blue, orange, green and red color graphs are the behaviour of the quantity $C$ for the value of $\alpha=0,0.01,0.0305$ (actual experimentally measured value) and 0.06 . In the Fig. $4 \mathrm{~b}$ we focus around the region where the value of the quantity $C$ is maximum. From Fig. 4 we observe that the maximum value of $C$ increase with the increase of the value of the mass hierarchy parameter $\alpha$

the expression for $C$, the maximum value of $C$ reduces to 2.16553 for $L_{1}=140.15 \mathrm{~km}$ and $\Delta L=1253.8 \mathrm{~km}$. So presence of $\delta_{\mathrm{CP}}$ actually enhances the maximum violation of LGI by an amount 0.00483 . This is a significant enhancement. Thus CP violation actually enhances the quantumness of the three-flavoured neutrino oscillations. It is worth mentioning that in the case of neutral kaon oscillations the presence of CP violation increases the maximum violation of LGI by an amount 0.00015 [2] which is a $0.008 \%$ enhancement, whereas here the increase is $0.24 \%$ i.e. a 30 -fold increase. Therefore, so far as LGI is concerned, the effect of the CP violation is much more in three-flavoured neutrino oscillations compared to neutral kaon oscillations. In Fig. 5 we focus around the region where the quantity $C$ takes its maximum value both with and without $\mathrm{CP}$ violation for experimentally obtained value of $\alpha=0.0305$.

\section{A proposal for experimental verification}

To test experimentally the maximum violation of LGI for three-flavoured neutrino oscillations the first thing necessary is the determination of the correlation function $C_{12}$. For this

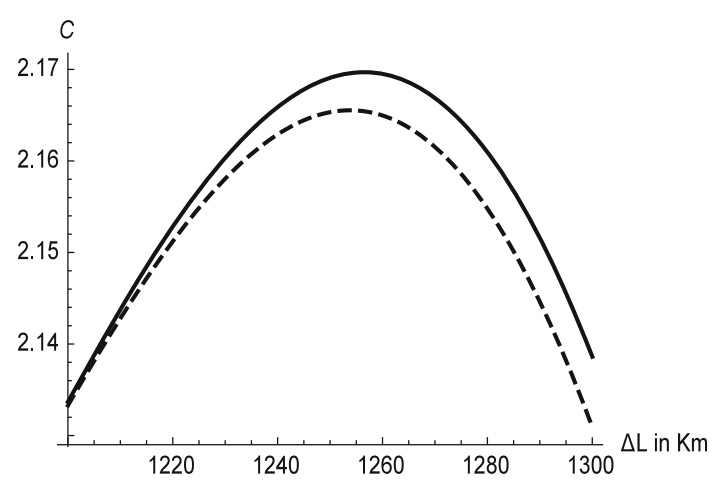

Fig. 5 Variation of the quantity $C$ as a function of $\Delta L$ in $\mathrm{km}$ with and without CP violation for $L_{1}=140.15 \mathrm{~km}$ is shown in Fig. 5 . Here we focus around the region where the quantity $C$ takes its maximum value both with and without $\mathrm{CP}$ violation. The solid curve is the behaviour of $C$ including $\mathrm{CP}$ violation and the dashed curve is the behaviour of $C$ without $\mathrm{CP}$ violation. Figure 5 tells that the presence of $\mathrm{CP}$ violation enhances the maximum QM violation of LGI

purpose the observable quantity $Q$ has to be measured at two different times $t_{1}$ and $t_{2}\left(t_{2}>t_{1}\right)$ or equivalently at two different base line lengths $L_{1}$ and $L_{2}$ where $L_{2}>L_{1}$. As already mentioned $Q$ takes the value +1 when the system is found in the electron-neutrino flavour state. Otherwise $Q$ takes the value -1 . So

$$
\begin{aligned}
C_{12}= & \mathscr{P}_{++}\left(L_{1}, L_{2}\right)-\mathscr{P}_{+-}\left(L_{1}, L_{2}\right)-\mathscr{P}_{-+}\left(L_{1}, L_{2}\right) \\
& +\mathscr{P}_{--}\left(L_{1}, L_{2}\right),
\end{aligned}
$$

where $\mathscr{P}_{++}\left(L_{1}, L_{2}\right)=P_{v_{e}, v_{e}}\left(L_{1}, L_{2}\right)$ is the joint probability of finding the system in the electron-neutrino flavour state at both the distances $L_{1}$ and $L_{2}$. Similar arguments hold for the other 3 joint probabilities:

$$
\begin{aligned}
& \mathscr{P}_{+-}\left(L_{1}, L_{2}\right)=P_{v_{e}, v_{\mu}}\left(L_{1}, L_{2}\right)+P_{v_{e}, v_{\tau}}\left(L_{1}, L_{2}\right), \\
& \mathscr{P}_{-+}\left(L_{1}, L_{2}\right)=P_{v_{\mu}, v_{e}}\left(L_{1}, L_{2}\right)+P_{v_{\tau}, v_{e}}\left(L_{1}, L_{2}\right), \\
& \mathscr{P}_{--}\left(L_{1}, L_{2}\right)=P_{v_{\mu}, v_{\mu}}\left(L_{1}, L_{2}\right)+P_{v_{\mu}, v_{\tau}}\left(L_{1}, L_{2}\right), \\
& \quad+P_{v_{\tau}, v_{\mu}}\left(L_{1}, L_{2}\right)+P_{v_{\tau}, v_{\tau}}\left(L_{1}, L_{2}\right) .
\end{aligned}
$$

Note that the scripted probabilities $\mathscr{P}$ are the ones that are actually measured. These are related to the theoretically calculated unscripted probabilities as shown above. This is necessitated by the fact that here more than one state can have the same value for the dichotomic variable $Q$.

It is to be noted that to experimentally verify the maximum violation of LGI the first measurement of $Q$ at length $L_{1}$ must satisfy NIM. Otherwise the measurement process will destroy the state of the system and measurement of $Q$ at the later length $L_{2}$ will be meaningless as the state has already been disturbed. This (NIM in the first measurement) can be ensured using the negative result measurement (NRM) [43] as follows.

Let the measuring set-up be arranged so that if the probe is triggered, $Q\left(L_{1}\right)=+1$, while if it is not triggered, $Q\left(L_{1}\right)=$ 
-1 . This ensures that while the untriggered probe provides information as regards the value of $Q$, there is no interaction occurring between the probe and the measured particle. So NIM is satisfied. Now use only the results of untriggered runs for which $Q\left(L_{1}\right)=-1$. Follow this by the measurement of $Q$ at $L_{2}$. These results can be used for determining the joint probabilities $P_{-+}\left(L_{1}, L_{2}\right)$ and $P_{--}\left(L_{1}, L_{2}\right)$. Similarly, for determining the other two joint probabilities $P_{+-}\left(L_{1}, L_{2}\right)$ and $P_{++}\left(L_{1}, L_{2}\right)$ occurring in $C_{12}$, the measuring set-up can be inverted so that a value of $Q\left(L_{1}\right)=-1$ triggers the probe, while for $Q\left(L_{1}\right)=+1$ it does not. In this way, one can determine $C_{12}$ and all the two-time correlation functions occurring in LGI by ensuring NIM through the use of the NRM procedure for the first measurement of any pair. Then one can calculate the total correlation $C$ using Eq. (1) and experimentally verify our results as regards the maximum violation of LGI in the case of three-flavour neutrino oscillations.

\section{Concluding remarks}

In this work we have investigated the violation of the LGI in the case of three-flavoured neutrino oscillations. Our findings are as follows:

1. The maximum value of the correlation $C$ is 2.17036 for $L_{1}=140.15 \mathrm{~km}$ and $\Delta L=1255.7 \mathrm{~km}$.

2. The violation of the classical bound of $C$ given by LGI for three-flavoured neutrino oscillation is $8.5 \%$. Note that in the case of two-flavoured neutrino oscillations [2] this violation was $38 \%$. So the maximum violation of LGI in the case of three-flavour neutrino oscillations is significantly lower than the maximum violation for the two-state neutrino oscillation.

3. If we put $\theta_{13}=0$, the maximum value of $C$ is 2.07762 for $L_{1}=638 \mathrm{~km}$ and $\Delta L=1376.34 \mathrm{~km}$. This is much lower than (2.17036) which is obtained for the experimental value of $\theta_{13}=8.5^{\circ}$. So the presence of $\theta_{13}$ enhances the maximum violation of LGI by the amount 0.09274 i.e. $4.6 \%$. Increasing $\theta_{13}$ increases the maximum value of C (Fig. 3).

4. For the mass hierarchy parameter $\alpha=0$, i.e. $m_{1}=m_{2}$ the maximum value of $C$ is 2.09606 for $\Delta L=1252.74 \mathrm{~km}$. Note that although now $m_{1}=m_{2}$ the maximum bound of $C$ is greater than 2, i.e., we are still in the quantum domain. For two-state neutrino oscillations, [2] $m_{1}=$ $m_{2}$ implied that the maximum value of $C$ is 2 , i.e. the classical domain. $\alpha=0.0305$ increases the maximum value of the quantity $C$ by $3.7 \%$ as shown in Fig. 4 .

5. If $\delta_{\mathrm{CP}}=0$ in the expression for $C$, the maximum value of $C$ reduces to 2.16553 for $L_{1}=140.15 \mathrm{~km}$ and $\Delta L=1253.8 \mathrm{~km}$. So the presence of a CP-violating phase parameter actually enhances the maximum violation of LGI by an amount 0.00483 which is $0.24 \%$, a significant enhancement (Fig. 5). Compare this to the case of neutral kaon oscillations where including $\mathrm{CP}$ violation increased the maximum violation of LGI by $0.008 \%$ [2].

Acknowledgements Animesh Sinha Roy thanks UGC-CSIR for providing a Research Fellowship Sr. No. 2061151173 under which this work was done.

Open Access This article is distributed under the terms of the Creative Commons Attribution 4.0 International License (http://creativecomm ons.org/licenses/by/4.0/), which permits unrestricted use, distribution, and reproduction in any medium, provided you give appropriate credit to the original author(s) and the source, provide a link to the Creative Commons license, and indicate if changes were made.

Funded by SCOAP ${ }^{3}$.

\section{Appendix}

The exact expression of the correlation function $C_{12}$ is given by

$$
\begin{aligned}
& C_{12}=P_{v_{e}, v_{e}}\left(L_{1}, L_{2}\right)-P_{v_{e}, v_{\mu}}\left(L_{1}, L_{2}\right)-P_{v_{e}, v_{\tau}}\left(L_{1}, L_{2}\right) \\
& -P_{v_{\mu}, v_{e}}\left(L_{1}, L_{2}\right)+P_{v_{\mu}, v_{\mu}}\left(L_{1}, L_{2}\right)+P_{v_{\mu}, v_{\tau}}\left(L_{1}, L_{2}\right) \\
& -P_{v_{\tau}, v_{e}}\left(L_{1}, L_{2}\right)+P_{v_{\tau}, v_{\mu}}\left(L_{1}, L_{2}\right)+P_{v_{\tau}, v_{\tau}}\left(L_{1}, L_{2}\right) \\
& =\left[1-\alpha^{2} \sin ^{2} 2 \theta_{12} \frac{\sin ^{2}\left(\frac{V L_{1}}{2}\right)}{\left(\frac{2 E V}{\Delta m_{31}^{2}}\right)^{2}}\right. \\
& \left.-4 s_{13}^{2} \frac{\sin ^{2}\left\{\left(\frac{2 E V}{\Delta m_{31}^{2}}-1\right) \frac{\Delta m_{31}^{2} L_{1}}{4 E}\right\}}{\left(\frac{2 E V}{\Delta m_{31}^{2}}-1\right)^{2}}\right] \\
& \times\left[1-2 \alpha^{2} \sin ^{2} 2 \theta_{12} \frac{\sin ^{2}\left(\frac{V\left(L_{2}-L_{1}\right)}{2}\right)}{\left(\frac{2 E V}{\Delta m_{31}^{2}}\right)^{2}}-8 s_{13}^{2}\right. \\
& \left.\times \frac{\sin ^{2}\left\{\left(\frac{2 E V}{\Delta m_{31}^{2}}-1\right) \frac{\Delta m_{31}^{2}\left(L_{2}-L_{1}\right)}{4 E}\right\}}{\left(\frac{2 E V}{\Delta m_{31}^{2}}-1\right)^{2}}\right] \\
& -\left[\alpha^{2} \sin ^{2} 2 \theta_{12} c_{23}^{2} \frac{\sin ^{2}\left(\frac{V L_{1}}{2}\right)}{\left(\frac{2 E V}{\Delta m_{31}^{2}}\right)^{2}}\right. \\
& +4 s_{13}^{2} s_{23}^{2} \frac{\sin ^{2}\left\{\left(\frac{2 E V}{\Delta m_{31}^{2}}-1\right) \frac{\Delta m_{31}^{2} L_{1}}{4 E}\right\}}{\left(\frac{2 E V}{\Delta m_{31}^{2}}-1\right)^{2}}+2 \alpha s_{13} \\
& \times \sin 2 \theta_{12} \sin 2 \theta_{23} \cos \left(\frac{\Delta m_{31}^{2} L_{1}}{4 E}-\delta_{\mathrm{CP}}\right) \\
& \left.\times \frac{\sin \left(\frac{V L_{1}}{2}\right)}{\left(\frac{2 E V}{\Delta m_{31}^{2}}\right)} \frac{\sin \left\{\left(\frac{2 E V}{\Delta m_{31}^{2}}-1\right) \frac{\Delta m_{31}^{2} L_{1}}{4 E}\right\}}{\left(\frac{2 E V}{\Delta m_{31}^{2}}-1\right)}\right]
\end{aligned}
$$




$$
\begin{aligned}
& \times\left[2 \alpha^{2} \sin ^{2} 2 \theta_{12} c_{23}^{2} \frac{\sin ^{2}\left(\frac{V\left(L_{2}-L_{1}\right)}{2}\right)}{\left(\frac{2 E V}{\Delta m_{31}^{2}}\right)^{2}}\right. \\
& +8 s_{13}^{2} s_{23}^{2} \frac{\sin ^{2}\left\{\left(\frac{2 E V}{\Delta m_{31}^{2}}-1\right) \frac{\Delta m_{31}^{2}\left(L_{2}-L_{1}\right)}{4 E}\right\}}{\left(\frac{2 E V}{\Delta m_{31}^{2}}-1\right)^{2}} \\
& +4 \alpha s_{13} \sin 2 \theta_{12} \sin 2 \theta_{23} \frac{\sin \left(\frac{V\left(L_{2}-L_{1}\right)}{2}\right)}{\left(\frac{2 E V}{\Delta m_{31}^{2}}\right)} \\
& \times \frac{\sin \left\{\left(\frac{2 E V}{\Delta m_{31}^{2}}-1\right) \frac{\Delta m_{31}^{2}\left(L_{2}-L_{1}\right)}{4 E}\right\}}{\left(\frac{2 E V}{\Delta m_{31}^{2}}-1\right)} \\
& \times\left\{\cos \left(\frac{\Delta m_{31}^{2}\left(L_{2}-L_{1}\right)}{4 E}-\delta_{\mathrm{CP}}\right)\right. \\
& \left.\left.-\sin \delta_{\mathrm{CP}} \sin \left(\frac{\Delta m_{31}^{2}\left(L_{2}-L_{1}\right)}{4 E}\right)\right\}-1\right] \\
& -\left[\alpha^{2} \sin ^{2} 2 \theta_{12} s_{23}^{2} \frac{\sin ^{2}\left(\frac{V L_{1}}{2}\right)}{\left(\frac{2 E V}{\Delta m_{31}^{2}}\right)^{2}}+4 s_{13}^{2} c_{23}^{2}\right. \\
& \times \frac{\sin ^{2}\left\{\left(\frac{2 E V}{\Delta m_{31}^{2}}-1\right) \frac{\Delta m_{31}^{2} L_{1}}{4 E}\right\}}{\left(\frac{2 E V}{\Delta m_{31}^{2}}-1\right)^{2}} \\
& -2 \alpha s_{13} \sin 2 \theta_{12} \sin 2 \theta_{23} \cos \left(\frac{\Delta m_{31}^{2} L_{1}}{4 E}-\delta_{\mathrm{CP}}\right) \\
& \left.\frac{\sin \left(\frac{V L_{1}}{2}\right)}{\left(\frac{2 E V}{\Delta m_{31}^{2}}\right)} \frac{\sin \left\{\left(\frac{2 E V}{\Delta m_{31}^{2}}-1\right) \frac{\Delta m_{31}^{2} L_{1}}{4 E}\right\}}{\left(\frac{2 E V}{\Delta m_{31}^{2}}-1\right)}\right] \\
& {\left[2 \alpha^{2} \sin ^{2} 2 \theta_{12} s_{23}^{2} \frac{\sin ^{2}\left(\frac{V\left(L_{2}-L_{1}\right)}{2}\right)}{\left(\frac{2 E V}{\Delta m_{31}^{2}}\right)^{2}}\right.} \\
& +8 s_{13}^{2} c_{23}^{2} \frac{\sin ^{2}\left\{\left(\frac{2 E V}{\Delta m_{31}^{2}}-1\right) \frac{\Delta m_{31}^{2}\left(L_{2}-L_{1}\right)}{4 E}\right\}}{\left(\frac{2 E V}{\Delta m_{31}^{2}}-1\right)^{2}} \\
& -4 \alpha s_{13} \sin 2 \theta_{12} \sin 2 \theta_{23} \frac{\sin \left(\frac{V\left(L_{2}-L_{1}\right)}{2}\right)}{\left(\frac{2 E V}{\Delta m_{31}^{2}}\right)} \\
& \times \frac{\sin \left\{\left(\frac{2 E V}{\Delta m_{31}^{2}}-1\right) \frac{\Delta m_{31}^{2}\left(L_{2}-L_{1}\right)}{4 E}\right\}}{\left(\frac{2 E V}{\Delta m_{31}^{2}}-1\right)} \\
& \left\{\cos \left(\frac{\Delta m_{31}^{2}\left(L_{2}-L_{1}\right)}{4 E}-\delta_{\mathrm{CP}}\right)\right. \\
& \left.\left.-\sin \delta_{\mathrm{CP}} \sin \left(\frac{\Delta m_{31}^{2}\left(L_{2}-L_{1}\right)}{4 E}\right)\right\}-1\right] \text {. }
\end{aligned}
$$

\section{References}

1. A.J. Leggett, A. Garg, Phys. Rev. Lett. 54, 857 (1985)

2. D. Gangopadhyay, D. Home, A. Sinha Roy, Phys. Rev. A 88, 022115 (2013)

3. J.A. Formaggio, D.I. Kaiser, M.M. Murskyj, T.E. Weiss, Phys. Rev. Lett. 117, 050402 (2016)

4. B. Pontecorvo, Zh Eksp, Teor. Fiz. 53, 1717 (1967)

5. B. Pontecorvo, Sov. Phys. JETP 26, 984 (1968)

6. V.N. Gribov, B. Pontecorvo, Phys. Lett. B 28, 493 (1969)

7. K. Kodama et al., Phys. Lett. B 504, 218 (2001)

8. M.C. Gonzalez-Garcia, M. Maltoni, Phys. Rep. 460, 1 (2008)

9. S.F. King, J. Phys. G Nucl. Part. Phys. 42, 123001 (2015)

10. J.S. Bell, Physics 1, 195 (1964)

11. A. Aspect, P. Grangier, G. Roger, Phys. Rev. Lett. 49, 91 (1982)

12. A. Aspect, J. Dalibard, G. Roger, Phys. Rev. Lett. 49, 1804 (1982)

13. W. Tittel, J. Brendel, H. Zbinden, N. Gisin, Phys. Rev. Lett. 81, 3563 (1998)

14. G. Weihs, T. Jennewein, C. Simon, H. Weinfurter, A. Zeilinger, Phys. Rev. Lett. 81, 5039 (1998)

15. M.A. Rowe, D. Kielpinski, V. Meyer, C.A. Sackett, W.M. Itano, C. Monroe, D.J. Wineland, Nature (London) 409, 791 (2001)

16. D. Salart, A. Baas, J.A.W. van Houwelingen, N. Gisin, H. Zbinden, Phys. Rev. Lett. 100, 220404 (2008)

17. C.H. van der Wal et al., Science 290, 773 (2000)

18. J.R. Friedman et al., Nature 406, 43 (2002)

19. R. Ruskov, A.N. Korotkov, A. Mizel, Phys. Rev. Lett. 96, 200404 (2006)

20. A.N. Jordan, A.N. Korotkov, M. Buttiker, Phys. Rev. Lett. 97, 026805 (2006)

21. N.S. Williams, A.N. Jordan, Phys. Rev. Lett. 100, 026804 (2008)

22. G.C. Knee et al., Nat. Commun. 3, 606 (2012)

23. M.E. Gossin et al., Proc. Natl Acad. Sci. 108, 1256 (2011)

24. A. Palacios-Laloy et al., Nat. Phys. 6, 442 (2010)

25. J. Dressel et al., Phys. Rev. Lett. 106, 040402 (2011)

26. A.M. Souza, I.S. Oliveira, R.S. Sarthour, New J. Phys. 13, 053023 (2011)

27. G. Waldherr et al., Phys. Rev. Lett. 107, 090401 (2011)

28. V. Athalye, S.S. Roy, T.S. Mahesh, Phys. Rev. Lett. 107, 130402 (2011)

29. C. Emary, Phys. Rev. A 87, 032106 (2013)

30. S.V. Moreira, A. Keller, T. Coudreau, P. Milman, Phys. Rev. A 92, $062132(2015)$

31. C. Budroni, C. Emary, Phys. Rev. Lett. 113, 050401 (2014)

32. J.J. Halliwell, Phys. Rev. A 93, 022123 (2016)

33. N. Lambert, K. Debnath, A.F. Kockum, G.C. Knee, W.J. Munro, F. Nori, Phys. Rev. A 94, 012105 (2016)

34. D. Gangopadhyay, A. Sinha Roy, Euro. Phys. Lett. 116, 40007 (2016)

35. C. Emary, N. Lambert, F. Nori, Rep. Prog. Phys. 77, 016001 (2014)

36. K. Hagiwara et al., Phys. Rev. D 66, 010001 (2002)

37. J. Beringer et al., Phys. Rev. D 86, 010001 (2012)

38. E.K. Akhmedov, R. Johansson, M. Lindner, T. Ohlsson, T. Schwetz, J. High Energy Phys. 04, 078 (2004)

39. M.C. Gonzalez-Garcia, M. Maltoni, T. Schwetz, J. High Energy Phys. 11, 052 (2014)

40. A. Nicolaidis, Phys. Lett. B 200, 553 (1988)

41. Q.Y. Liu, S.P. Mikheyev, A.Y. Smirnov, Phys. Lett. B 440, 319 (1998)

42. M. Freund, T. Ohlsson, Mod. Phys. Lett. A 15, 867 (2000)

43. D. Home, Curr. Sci. 109, 11 (2015) 